\title{
Thoracoscopic versus open resection for symptomatic congenital cystic adenomatoid malformations of the lung in neonates: a decade of experience
}

\author{
jintao zheng ${ }^{1}$, Huajian Tang ${ }^{1}$, Jiequan $\mathrm{Li}^{1}$, Huiyu Xu${ }^{1}$, Weidong Liu ${ }^{1}$, and Guoqing Liu ${ }^{1}$ \\ ${ }^{1}$ Southern Medical University
}

June 17, 2020

\begin{abstract}
Purpose: The purpose of this study is to present our surgical experience with symptomatic congenital cystic adenomatoid malformations (CCAMs) in neonates. Methods: The medical records of neonates who underwent surgery for symptomatic CCAM from 2010 to 2020 were retrospectively reviewed. Results: Of 24 cases, 10 involved open resection, and 14 involved thoracoscopic resection. Four cases of CCAMs located in the upper or middle lobes underwent lobectomy, and 20 cases underwent lung-preserving wedge resection in the lower lobe. Between the two groups, there was no statistically significant difference in related preoperative variables, including gestational age at birth, body weight, head circumference, lesion size, CVR, and age at operation $(\mathrm{P}>.05)$. The differences in intraoperative variables were statistically significant. The length of the surgical incision in the thoracoscopic resection group was significantly shorter than that in the open resection group (1.4 cm [1.3-1.8] vs $6.0 \mathrm{~cm}$ [5.0-8.0], $\mathrm{P}=.000)$, and there was significantly less operative blood loss (3 $\mathrm{ml}[1-6]$ vs $5 \mathrm{ml}[2-10], \mathrm{P}=.030)$, but the operation time was significantly longer (159 min [100-220] vs $110 \mathrm{~min}$ [70-170], $\mathrm{P}=.003)$. Regarding postoperative variables, ventilator days, duration of chest tube use and length of hospital stay were not statistically significant $(\mathrm{P}>.05)$. Conclusions: Thoracoscopic resection is a safe and favorable approach for symptomatic CCAM in neonates. It has minimal esthetic effects and does not increase the risk of surgical or postoperative complications. Lung-preserving resection is feasible for neonatal CCAM surgery.
\end{abstract}

\section{Introduction}

Cystic adenomatoid malformation (CCAM) is a fetal lung malformation. It has been reported that the incidence of CCAM is between 1:11,000 and 1:35,000 ${ }^{1}$. CCAM is characterized by benign malignancies or dysplastic lung tumors with excessive growth of terminal bronchioles and a decrease in the number of alveoli $^{2}$. The perinatal mortality rate associated with prenatally diagnosed CCAM varies widely, ranging from $9 \%$ to $49 \%^{3}$. This is because the clinical characteristics of CCAM vary greatly, from acute respiratory distress at birth to incidental asymptomatic lesions on a chest radiograph at any age. Whether asymptomatic CCAM requires surgery during the neonatal period is still controversial, but it is clear that surgery is the accepted standard of care for all symptomatic CCAMs, even in neonates ${ }^{4,5}$. Infection is the most common presenting symptom ${ }^{6}$. We carried out surgical treatment for symptomatic CCAM patients after the infection had adequately subsided. CCAM can be treated with open or thoracoscopic resection. To date, few reports have compared the outcomes of the two surgical methods for the treatment of CCAM in neonates. Therefore, the purpose of this study is to compare the preoperative, intraoperative, and postoperative variables of the two surgical procedures and to determine whether the two surgical methods have an effect on the outcome of CCAM.

\section{Methods}


The study design was a retrospective review of the data from 24 symptomatic neonates with CCAM who underwent thoracoscopic or open resection at the Foshan Women and Children Hospital Affiliated to Southern Medical University, from April 2010 to April 2020. The pathological results of all cases were confirmed to be CCAM or CCAM combined with pulmonary sequestration (PS). The medical records were reviewed for preoperative, intraoperative and postoperative variables. Preoperative variables included gestational age at birth, sex, body weight, head circumference, location of the CCAM, lesion size, cystic adenomatoid malformation volume ratio $(\mathrm{CVR})^{7}$, respiratory symptoms, associated anomalies and age at operation. Intraoperative variables included length od surgical incision, operation time and operative blood loss. Postoperative variables included postoperative ventilator days, duration of chest tube use, length of postoperative hospital stay and postoperative complications.

All cases were performed with preoperative CT or MRI scans and 3D reconstruction to better determine the range of the lesion (Fig. 1-A). Surgery was performed after the infection had adequately subsided. Central venous and bladder catheters were placed.

For the thoracoscopic resection procedure, general anesthesia, tracheal intubation and nonsingle lung ventilation were used. The patient was placed in a semiprone position with the affected side elevated to more easily expose the lesion. The first 5 -mm trocar for thoracoscopy (5-mm 300, Olympus) was placed at the tip of the scapula. The second 5-mm trocar was inserted at the posterior axillary line, and the third 3-mm trocar was inserted at the interscapular region. Both trocars were parallel to the tip of the scapula. (Fig. 1-B) All trocars were sutured and fixed to prevent them from being pulled out. Carbon dioxide pressure was $3 \mathrm{mmHg}$ to $5 \mathrm{mmHg}$. Thoracoscopic lobectomy or wedge resection was performed according to the size of the lesion (Fig. 1-C). An ultrasonically activated scalpel was used for wedge resection, and the lung wound was sutured with 4-0 absorbable thread. Hemo-locks were used to clip the main blood vessel and main bronchus during lobectomy. The chest tube was conventionally placed.

Statistical analyses were performed using SPSS Statistics (version 12.0.0.0). The Mann-Whitney U test was used to compare continuous variables with nonparametric variables. Fisher's exact test and $2 \times 2$ contingency tables were used to compare categorical variables. Statistical significance was defined as a $\mathrm{P}$ value of less than .05. This study was approved by Medical Ethics Committee of Foshan Women and Children Hospital Affiliated to Southern Medical University (FSFY-MEC-2020-031).

\section{Results}

Twenty-four neonates with symptomatic CCAM underwent thoracoscopic resection or open resection at the Foshan Women and Children Hospital Affiliated to Southern Medical University, from April 2010 to April 2020. Ten patients underwent open resection before April 2017, whereas 14 underwent thoracoscopic resection after that time.

\subsection{Patient population and characteristics}

Of the 24 patients, 14 were male and 10 were female. Twenty-three patients were diagnosed with prenatal sonography. The most common respiratory symptoms were pneumonia, respiratory distress and shortness of breath. All of the patients had suffered from neonatal pneumonia; two patients had pneumonia with respiratory distress, and eight had pneumonia with shortness of breath. Two patients had mediastinal deviation due to large lung lesions. Four patients with CCAM located in the upper or middle lobes underwent lobectomy, and 20 underwent lung-preserving wedge resection in the lower lobe, regardless of whether the lesion was on the left or right side. The final pathological result was 20 cases of CCAM and 4 cases of CCAM with PS. One patient was found to have congenital chylothorax after the operation and healed after sufficient drainage. One patient had postoperative complications of a air leaks and healed spontaneously after conservative treatment. Details of the patient population and the characteristics of the thoracoscopic resection and open resection groups are shown in Table 1 . Some variables were statistically analyzed, and there was no statistically significant difference between the two groups.

The median follow-up time was 25 months (range, 1-47 months), and none of the patients experienced 
postoperative recurrence, shortness of breath, difficulty breathing after activity, or repeated pneumonia. One patient who was treated with open resection had a slightly sunken chest while breathing.

\subsection{Results of the comparison of related variables: thoracoscopic versus open resection}

There were no statistically significant differences between the thoracoscopic resection group and the open resection group in the related preoperative variables (median [range]), including gestational age at birth (39.4 $\mathrm{w}$ [34.1-41.1] vs $39.3 \mathrm{w}$ [35.5-41.4], $\mathrm{P}=.977)$, body weight (3260 g [2100-4270] vs $3240 \mathrm{~g}$ [2760-3860], $\mathrm{P}=.838$ ), head circumference $(33 \mathrm{~cm}$ [32-37] vs $34 \mathrm{~cm}$ [31-34], $\mathrm{P}=.785)$, lesion size $\left(24.00 \mathrm{~cm}^{3}[12.00-63.00]\right.$ vs 34.85 $\mathrm{cm}^{3}$ [8.00-125.00], $\left.\mathrm{P}=.319\right)$, CVR (0.37 [0.18-1.00] vs 0.55 [0.13-1.92], $\left.\mathrm{P}=.266\right)$, and age at operation $(10 \mathrm{~d}$ [5-21] vs $11 \mathrm{~d}[7-17], \mathrm{P}=.442$ ) (Table 2). The related intraoperative variables showed statistically significant differences between the groups. The length of the surgical incision in the thoracoscopic resection group was significantly shorter than that in the open resection group $(1.4 \mathrm{~cm}[1.3-1.8]$ vs $6.0 \mathrm{~cm}$ [5.0-8.0], $\mathrm{P}=.000)$, and there was significantly less blood loss in the thoracoscopic resection group (3 $\mathrm{ml}$ [1-6] vs $5 \mathrm{ml}$ [2-10], $\mathrm{P}=.030$ ), but their operation time was significantly longer (159 min [100-220] vs 110 min [70-170], $\mathrm{P}=.003$ ) (Fig. 2).Regarding the related postoperative variables, the postoperative ventilator days $(3 \mathrm{~d}[1-6]$ vs $2 \mathrm{~d}$ [1-3], $\mathrm{P}=.159)$, duration of chest tube use ( $8 \mathrm{~d}$ [4-22] vs $6 \mathrm{~d}$ [4-15], $\mathrm{P}=.052)$ and length of postoperative hospital stay (12 d [10-25] vs 12.5 d [9-16], $\mathrm{P}=.425)$ did not significantly differ between the two groups.

\section{Discussion}

In light of improvements in antenatal ultrasound diagnosis, an increased number of fetuses are diagnosed with CCAM in utero. In this study, 23 patients were diagnosed with CCAM through prenatal sonography, with a diagnosis rate of up to $96 \%(23 / 24)$. Most CCAMs are not associated with severe respiratory symptoms after birth, but approximately $10 \%$ of lung lesions, including CCAMs, will present with symptoms during the neonatal period ${ }^{8}$. Infection is the most common presenting symptom, as indicated by the patients in this study. Other symptoms include shortness of breath, respiratory distress, and mediastinal deviation. The optimal timing for the resection of asymptomatic CCAM remains controversial, but surgery is the accepted standard for all symptomatic CCAMs ${ }^{9,10}$, and thoracoscopic operations are safe and feasible, even in neonates ${ }^{11,12}$. After the infection subsided sufficiently, we performed surgical treatment of symptomatic CCAM patients.

This study shows that both thoracoscopic and open resection for symptomatic CCAM have achieved good clinical outcomes in the neonatal period. When the two surgical methods are compared under the same preoperative conditions, thoracoscopic resection results in a shorter surgical incision and less operative blood loss, but the operation time is longer. Therefore, thoracoscopic surgery achieved an esthetic effect consistent with minimally invasive surgery. These clinical results are similar to those described in the literature ${ }^{4,13,14}$. In our experience, neonatal CCAM has a clear boundary with normal lung tissue and no adhesions caused by repeated infections. The blood vessels and bronchial tubes are relatively small and can be directly cut off by an ultrasonically activated scalpel. These characteristics are conducive to thoracoscopic resection. Thoracoscopic resection of CCAM has one unfavorable factor: the operation space is small, making it difficult to perform this operation during the neonatal period. Prior to this, we performed thoracoscopic operations for congenital diaphragmatic hernia and extralobar pulmonary sequestration to improve our skills. In addition, when suturing is difficult, a 3-mm trocar can also be added to assist. If blood oxygen maintenance is unstable during the operation, it is necessary to temporarily stop the operation and stop the artificial pneumothorax.

The extent of surgical resection in the management of CCAM also remains controversial ${ }^{15}$. Muller, CO et al. ${ }^{16}$ state that adequate treatment of CCAM in children requires lobectomy because of poor sensitivity and very poor negative predictive value of preoperative CT for determining distal adjacent lesions. Laberge JM et al ${ }^{6}$ think that Lobectomy is recommended in order to prevent postoperative air leaks, residual disease, and perhaps reduce the risk of some later malignancies. Fascetti-Leon, F. et al. ${ }^{17}$ state that lung-sparing surgery for congenital lung malformations is a safe and effective method of lung parenchymal preservation in pediatric patients. If accurately planned in selected patients, lung-sparing surgery does not carry a higher risk of residual disease and recurrence than traditional lobectomy. Kim, HK et al. ${ }^{18}$ report that the early and 
late outcomes were excellent even after parenchyma-saving resection in patients with CCAM, and suggest that parenchyma-saving resection can be safely performed in selected patients with a well-confined CCAM lesion and thereby avoiding lobectomy.

In this study, twenty patients underwent a lung-preserving wedge resection strategy for the treatment of CCAM of the lower lobe. Four patients underwent lobectomy because the CCAM almost completely occupied the upper or middle lobe. One early patient had the postoperative complication of an air leaks, which was cured by continuous thoracic drainage. We think that this complication was caused by poor wound healing rather than a problem with the lung preservation strategy because there was no recurrence in the postoperative follow-up. It may also have been related to unskilled suturing under thoracoscopy at an early stage of the procedure, but the complication rate was not statistically significantly different from that of open resection $(\mathrm{P}=1)$.

In conclusion, based on the data and follow-up results of this study, we believe that thoracoscopic resection is a safe and favorable approach for treating symptomatic CCAM of the lung in neonates. Compared with open resection, thoracoscopic surgery has minimal esthetic effects and does not increase the risk of surgical or postoperative complications. The lung-preserving resection strategy is feasible for neonatal CCAM patients. However, the study results are limited because of the small number of cases. In the next step, we will continue to perform long-term follow-up and evaluate the respiratory function of these patients.

\section{Acknowledgments}

This work was supported by a project funded by the China Postdoctoral Science Foundation (Postdoc no. 241006) and Science and Technology Planning Project 2019 of the Bureau of Science and Technology of Foshan City (no. 1920001000338).

\section{Competing Interests}

The authors declare that there are no competing interests regarding the publication of this paper.

\section{References}

1. Gornall AS, Budd JL, Draper ES, Konje JC, Kurinczuk JJ. Congenital cystic adenomatoid malformation: accuracy of prenatal diagnosis, prevalence and outcome in a general population. Prenat Diagn.2003;23(12):997-1002.

2. Stocker JT, Madewell JE, Drake RM. Congenital cystic adenomatoid malformation of the lung. Classification and morphologic spectrum.Hum Pathol. 1977;8(2):155-171.

3. Di Prima FA, Bellia A, Inclimona G, Grasso F, Teresa M, Cassaro MN. Antenatally diagnosed congenital cystic adenomatoid malformations (CCAM): Research Review. J Prenat Med. 2012;6(2):22-30.

4. Kaneko K, Ono Y, Tainaka T, Sumida W, Kawai Y, Ando H. Thoracoscopic lobectomy for congenital cystic lung diseases in neonates and small infants. Pediatr Surg Int. 2010;26(4):361-365.

5 . Eber E. Antenatal diagnosis of congenital thoracic malformations: early surgery, late surgery, or no surgery? Semin Respir Crit Care Med. 2007;28(3):355-366.

6. Laberge JM, Puligandla P, Flageole H. Asymptomatic congenital lung malformations. Semin Pediatr Surg. 2005;14(1):16-33.

7. Crombleholme TM, Coleman B, Hedrick H, et al. Cystic adenomatoid malformation volume ratio predicts outcome in prenatally diagnosed cystic adenomatoid malformation of the lung. J Pediatr Surg.2002;37(3):331338.

8. Singh R, Davenport M. The argument for operative approach to asymptomatic lung lesions. Semin Pediatr Surg.2015;24(4):187-195. 
9. Jelin EB, O'Hare EM, Jancelewicz T, Nasr I, Boss E, Rhee DS. Optimal timing for elective resection of asymptomatic congenital pulmonary airway malformations. J Pediatr Surg. 2018;53(5):1001-1005.

10. Duron V, Zenilman A, Griggs C, et al. Asymptomatic Congenital Lung Malformations: Timing of Resection Does Not Affect Adverse Surgical Outcomes. Front Pediatr. 2020;8:35.

11. Nasr A, Bass J. Thoracoscopic vs open resection of congenital lung lesions: a meta-analysis. J Pediatr Surg. 2012;47(5):857-861.

12. Rothenberg SS, Middlesworth W, Kadennhe-Chiweshe A, et al. Two decades of experience with thoracoscopic lobectomy in infants and children: standardizing techniques for advanced thoracoscopic surgery. $J$ Laparoendosc Adv Surg Tech A. 2015;25(5):423-428.

13. Vu LT, Farmer DL, Nobuhara KK, Miniati D, Lee H. Thoracoscopic versus open resection for congenital cystic adenomatoid malformations of the lung. J Pediatr Surg. 2008;43(1):35-39.

14. Adams S, Jobson M, Sangnawakij P, et al. Does thoracoscopy have advantages over open surgery for asymptomatic congenital lung malformations? An analysis of 1626 resections. J Pediatr Surg.2017;52(2):247251.

15. Annunziata F, Bush A, Borgia F, et al. Congenital Lung Malformations: Unresolved Issues and Unanswered Questions. Front Pediatr. 2019;7:239.

16. Muller CO, Berrebi D, Kheniche A, Bonnard A. Is radical lobectomy required in congenital cystic adenomatoid malformation? J Pediatr Surg. 2012;47(4):642-645.

17. Fascetti-Leon F, Gobbi D, Pavia SV, et al. Sparing-lung surgery for the treatment of congenital lung malformations. J Pediatr Surg.2013;48(7):1476-1480.

18. Kim HK, Choi YS, Kim K, et al. Treatment of congenital cystic adenomatoid malformation: should lobectomy always be performed? Ann Thorac Surg. 2008;86(1):249-253.

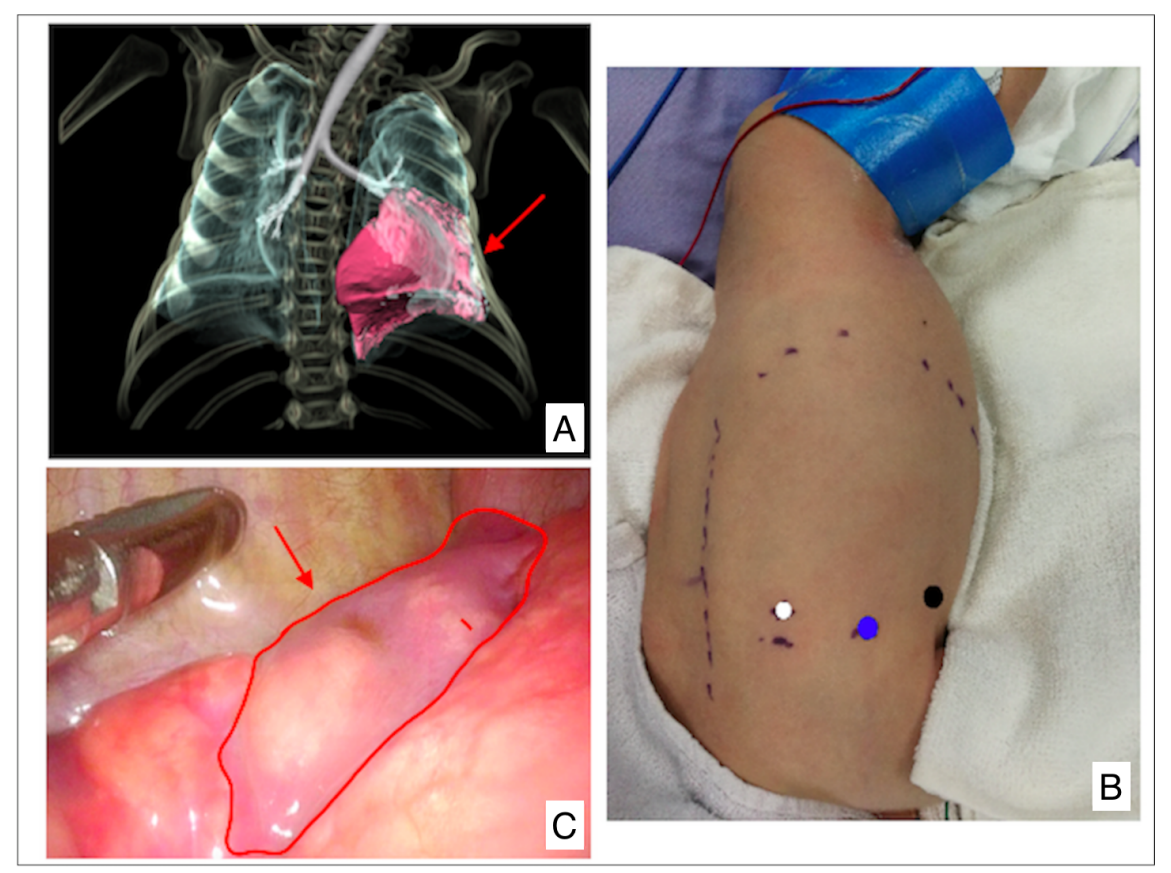

\section{Hosted file}


Table 1 Patient population and characteristics.docx available at https://authorea.com/users/ 334453/articles/460427-thoracoscopic-versus-open-resection-for-symptomatic-congenitalcystic-adenomatoid-malformations-of-the-lung-in-neonates-a-decade-of-experience

\section{Hosted file}

Table 2 Comparison of related variables- thoracoscopic resection versus open resection .docx available at https://authorea.com/users/334453/articles/460427-thoracoscopic-versus-openresection-for-symptomatic-congenital-cystic-adenomatoid-malformations-of-the-lung-inneonates-a-decade-of-experience
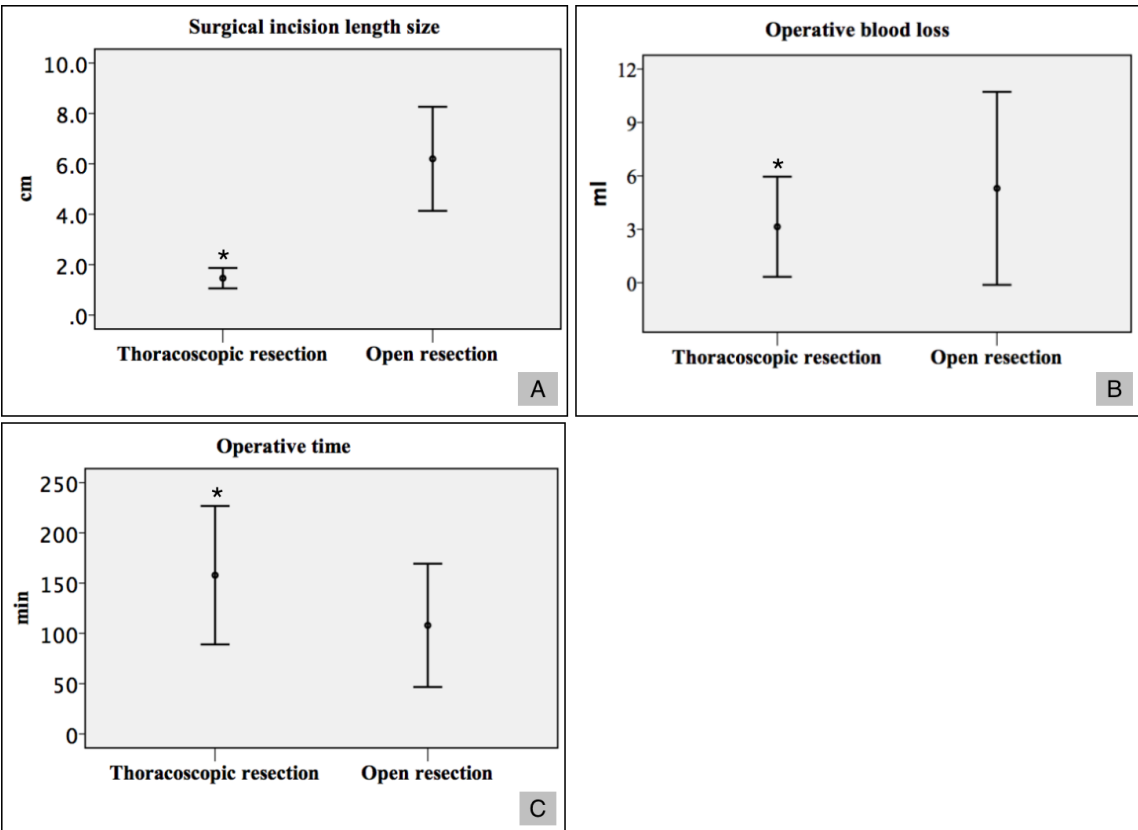